Results: As compared with those of HCs, patients with SLE had lower absolute numbers of total $\mathrm{T}, \mathrm{NK}$, and $\mathrm{CD} 4^{+} \mathrm{T}$ but higher proportions of all lymphocyte subpopulations except NK, CD4+T cells $(P<0.001)$ (Figure $1 \mathrm{~A}, \mathrm{C})$. Notably, the absolute numbers and proportions of Tregs as well as Th1 in $\mathrm{CD} 4^{+} \mathrm{T}$ subsets were decreased $(P<0.05)$ (Figure $1 \mathrm{~B}, \mathrm{D})$. Further, there was a significant increase in the ratio of Teffs/Tregs such as Th1/Tregs, Th2/Tregs and Th17/Tregs $(P<0.05)$ (Figure $1 \mathrm{E}$ ). After receiving immunoregulatory combination therapies, the absolute numbers and proportions of $\mathrm{T}, \mathrm{NK}, \mathrm{CD} 4^{+} \mathrm{T}$, and $\mathrm{CD}^{+}{ }^{+} \mathrm{T}$ were increased, while the proportion of $\mathrm{B}$ cells was decreased (Figure $2 \mathrm{~A}, \mathrm{C}$ ); the absolute numbers of most $C D 4^{+} \mathrm{T}$ subsets as well as the proportions of only Th1 and Tregs were significantly increased $(P<0.001)$ (Figure $2 \mathrm{~B}, \mathrm{D})$. The ratios of Th1/Th2 and Th1/Tregs increased while that of Th17/Tregs and Th2/Tregs decreased $(P<0.01)$ (Figure $2 \mathrm{E}$ ).

Conclusion: Quantitative and functional alterations of peripheral lymphocyte subsets, especially reduced Tregs, play crucial roles in the pathogenesis of the patients. Immunoregulatory combination therapies mainly promote the proliferation and functional recovery of Tregs to rebalance pro- and anti-inflammatory $T$ cells in patients with SLE for patients' symptoms remission.

References:

[1] Sharabi A, Tsokos GC. T cell metabolism: new insights in systemic lupus erythematosus pathogenesis and therapy. Nat Rev Rheumatol 2020 doi: 10.1038/s41584-019-0356-x [published Online First: 2020/01/18]

[2] Durcan L, O'Dwyer T, Petri M. Management strategies and future directions for systemic lupus erythematosus in adults. Lancet 2019;393(10188):233243. doi: 10.1016/S0140-6736(19)30237-5 [published Online First: 2019/06/11]

[3] Spolski R, Li P, Leonard WJ. Biology and regulation of IL-2: from molecular mechanisms to human therapy. Nat Rev Immunol 2018;18(10):648-59. doi: 10.1038/s41577-018-0046-y [published Online First: 2018/08/10]
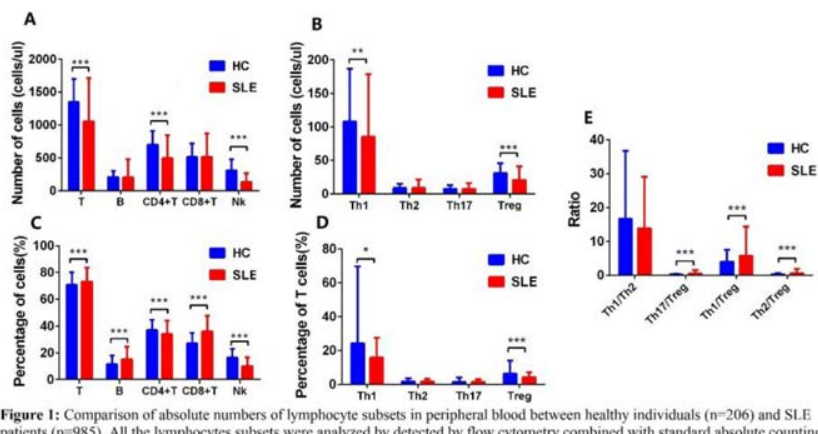
patients $(\mathrm{n}=985$ ). All the lymphocytes subsets were analyzed by detected by flow cytometry combined with standard absolute counting
beads. Data were presented as mean $\pm \mathrm{SD}$ and statistical analysis was determined by two-tailed unpaired 1 - - est. $* \mathrm{P}<0.05, * \cdots \mathrm{P}<0.01$,

Acknowledgments : None.

Disclosure of Interests: None declared

DOI: 10.1136/annrheumdis-2020-eular.1867

\section{SAT0182 THE ASSOCIATION BETWEEN AUTOANTIBODIES AND RITUXIMAB RESPONSE IN SYSTEMIC LUPUS ERYTHEMATOSUS}

D. Li $i^{1}$, H. Lu', J. Dunphy ${ }^{2}$, T. Smith ${ }^{1}$, E. Vital ${ }^{3,4}$, I. N. Bruce ${ }^{5}$, N. Mchugh ${ }^{1} .{ }^{1}$ The University of Bath, Pharmacy and Pharmacology, Bath, United Kingdom; ${ }^{2}$ Royal United Hospital Bath NHS Foundation Trust, Bath, United Kingdom;

${ }^{3}$ The University of Leeds, Leeds Institute of Rheumatic and Musculoskeletal Disease, Leeds, United Kingdom; ${ }^{4}$ Leeds Teaching Hospitals NHS Trust, NIHR Leeds Biomedical Research Centre, Leeds, United Kingdom; ${ }^{5}$ The University of Manchester, Centre for Epidemiology Versus Arthritis, Faculty of Biology Medicine and Health, Manchester, United Kingdom

Background: Systemic Lupus Erythematosus (SLE) is clinically and immunologically heterogeneous with a variable response to treatment. MASTERPLANS is an MRC-funded consortium that seeks to identify immunophenotypic subgroups of patients that predict response to therapy. Autoantibody profiles can differentiate subgroups of patients and have potential to predict response to treatment.

Objectives: To determine whether known and novel autoantibodies are associated with response to rituximab (RTX), and analyse the association between these antibodies and disease involvement in various organ systems.

Methods: Serum was obtained from 224 SLE patients in the BILAG Biologics Registry who received rituximab according to NHS England criteria (2). Patients were recruited if they were starting a first cycle of rituximab for active SLE (BILAG A or 2xBILAG B) despite previous cyclophosphamide or mycophenolate mofetil.
Evidence of any single organ system involvement previous or current was taken as having a BILAG score of A-D but not E. Disease activity was measured using BILAG-2004. Clinical response was defined as improvement by $>=1$ grade in active BILAG-2004 systems with no worsening in other systems. Autoantibodies were measured by immunoprecipitation of proteins by sera from ${ }^{35} \mathrm{~S}$-labelled $\mathrm{K} 562$ cell lines, followed by SDS-PAGE separation and autoradiography. Autoantibodies not able to be detected by this technique (anti-Ro52, anti-dsDNA and $\mathrm{ACL}$ ) were measured by ELISA. Autoantibody data was analysed in IBM SPSS and GraphPad Prism v8.2. Association between autoantibodies and RTX response was analysed using binary logistic interaction terms and Pearson's Chi-Square test.

Results: Of the 224 patients ( 201 female, 23 male, median age 40 years) the most common system involvement from the 9 BILAG domains was musculoskeletal (164 patients) and the least ophthalmic (11 patients). Patients with anti-Ro52 and antiU1RNP/Sm had more frequent involvement of mucocutaneous $(p<0.036, p<0.012)$ and musculoskeletal domains ( $p<0.015$ for U1RNP) respectively.

There were 136 patients with sufficient data to define as either responders $(n=67)$ or non-responders $(n=69)$ to RTX at 6 months. RTX responders had a higher frequency of anti-U1RNP/Sm compared to non-responders (Figure 1). Further Pearson's Chi-Square analysis showed a significant association between presence of anti-U1RNP/Sm and better response to RTX $(p<0.018)$.
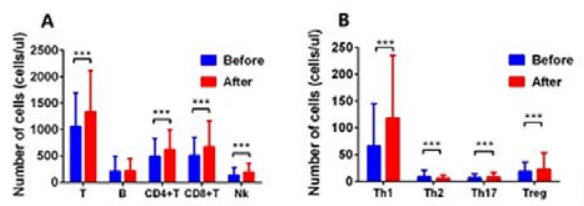

C
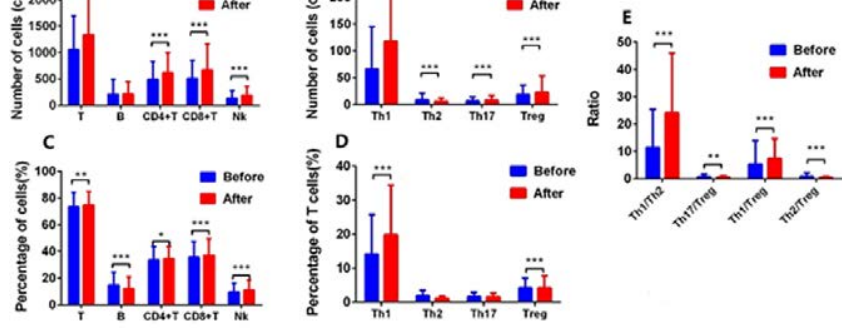

Figure 2: Changes of peripheral lymphocyle subsets after immunomodulatory combination therapy $(\mathrm{n}=795)$. Data were

Conclusion: Our findings suggest that the presence of U1RNP/Sm autoantibodies in a cohort of patients who have received treatment with RTX is associated with more frequent musculoskeletal and mucocutaneous involvement and predicts a more favourable response to treatment.

Acknowledgments : Funded by a grant from the Medical Research Council, grant number MR/M01665X/1. BILAG BR has been funded by unrestricted educational donations from Roche, GSK and LUPUS UK. Part-funded by a grant from LUPUS UK Disclosure of Interests: : Danyang Li: None declared, Hui Lu: None declared, Juliet Dunphy: None declared, Theresa Smith: None declared, Edward Vital Grant/ research support from: AstraZeneca, Roche/Genentech, and Sandoz, Consultant of: AstraZeneca, GSK, Roche/Genentech, and Sandoz, Speakers bureau: Becton Dickinson and GSK, lan N. Bruce Grant/research support from: Genzyme Sanofi, GSK, and UCB, Consultant of: Eli Lilly, AstraZeneca, UCB, Iltoo, and Merck Serono, Speakers bureau: UCB, Neil McHugh: None declared DOI: 10.1136/annrheumdis-2020-eular.4284

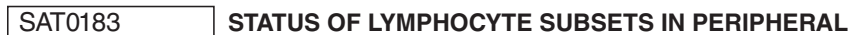 BLOOD OF PATIENTS WITH PRIMARY SJÖGREN'S SYNDROME AND THEIR CHANGES AFTER RECEIVING OUR NEW IMMUNOREGULATORY COMBINATION THERAPY: A RETROSPECTIVE CROSS-SECTIONAL STUDY}

J. Y. Yang ${ }^{1}$, S. X. Zhang ${ }^{2}$, L. $\mathrm{Hao}^{2}$, Q. Y. Su${ }^{1}$, J. Bai ${ }^{1}$, S. J. Guo ${ }^{1}$, J. Luo ${ }^{2}$, G. Y. Liu' ${ }^{2}, \mathrm{C} . \mathrm{GaO}^{3}, \mathrm{X} . \mathrm{Li}^{2}{ }^{1}{ }^{1}$ Shanxi Medical University, Taiyuan, China; ${ }^{2}$ The Second Hospital of Shanxi Medical University, Taiyuan, China; ${ }^{3}$ Brigham and Women's Hospital, Harvard Medical School, Boston, United States of America

Background: Primary Sjögren's syndrome (pSS) is a chronic inflammatory autoimmune disease mainly involving exocrine glands and involving multiple organs and systems ${ }^{1}$. Recent studies have reported that peripheral lymphocyte subsets such as Th1, Th2, Th17, and regulatory cells (Tregs), have been implicated in the pathogenesis of $\mathrm{pSS}^{2}$. However, the detailed statuses of lymphocyte subsets of pSS patients remain to be clearly evaluate and effects of immunomodulatory therapies on the lymphocyte subsets are unknown. 
Objectives: To explore the pathogenesis and evaluate the therapeutic effect of immunomodulatory drugs (IMiDs) by comparing the changes of lymphocyte subsets in peripheral blood (PB) before and after treatment.

Methods: This study included 1,221 pSS patients and 206 healthy controls (HCs). Among these patient, 759 patients were received our new immunoregulatory therapies such as low-dose interleukin-2, rapamycin, metformin, retinoic acid etc. The absolute numbers of $\mathrm{T}, \mathrm{B}, \mathrm{NK}, \mathrm{CD} 4^{+} \mathrm{T}, \mathrm{CD} 8^{+} \mathrm{T}, \mathrm{Th} 1, \mathrm{Th} 2, \mathrm{Th} 17$ and Tregs in PB of these subjects were detected by flow cytometry combined with standard absolute counting beads. Data were expressed as mean \pm standard deviation to the distribution. Independent-samples T test and paired-samples T test were applied. $P$ value $<0.05$ were considered statistically significant.

Results: The absolute numbers of circulating Tregs as well as T, NK cells in pSS patients were significantly lower than those of HCs $(P<0.05)$. After immunoregulatory combination treatments, the number of Tregs was significantly increased $(P$ $<0.05)$. Though the absolute numbers of $\mathrm{T}, \mathrm{NK}, \mathrm{CD} 4^{+} \mathrm{T}, \mathrm{CD} 8^{+} \mathrm{T}$, Th1, and Th17 cells were also increased to some degree $(P<0.05)$, the increased amount of Tregs was much more than other cells, resulting a new balance between pro- and anti- inflammatory lymphocyte homeostasis.

Conclusion: The decrease of peripheral Tregs played an important role in the pathogenesis of primary Sjögren's syndrome. Immunoregulatory combination therapies promoted the increase of Tregs and might help for the recovery of pSS.

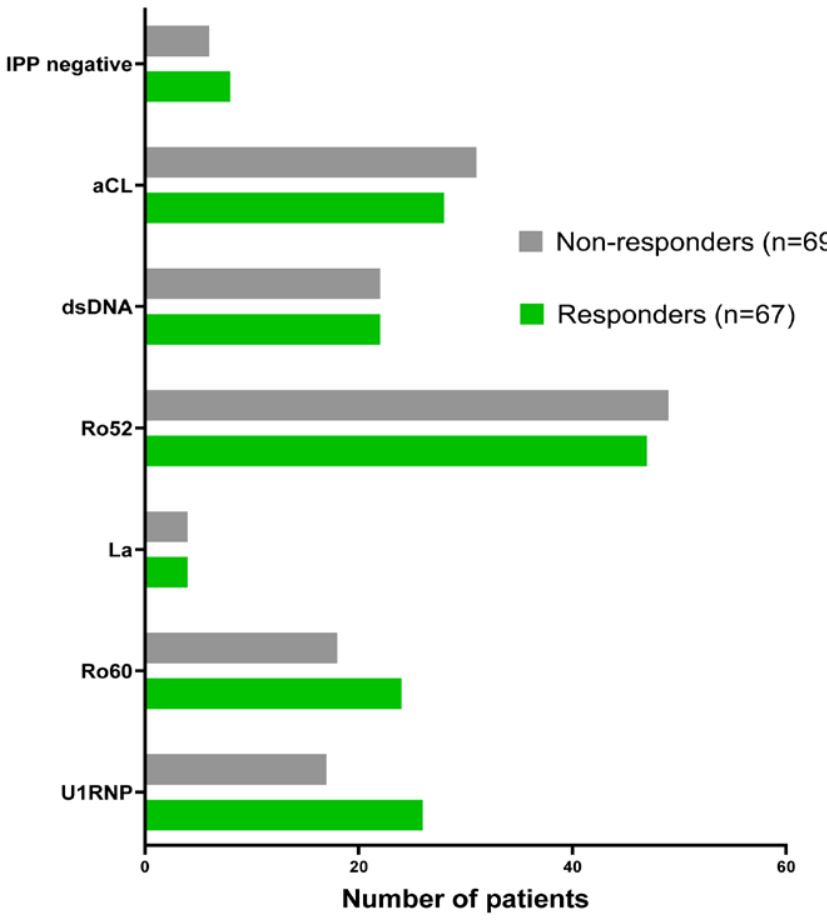

Figure 1
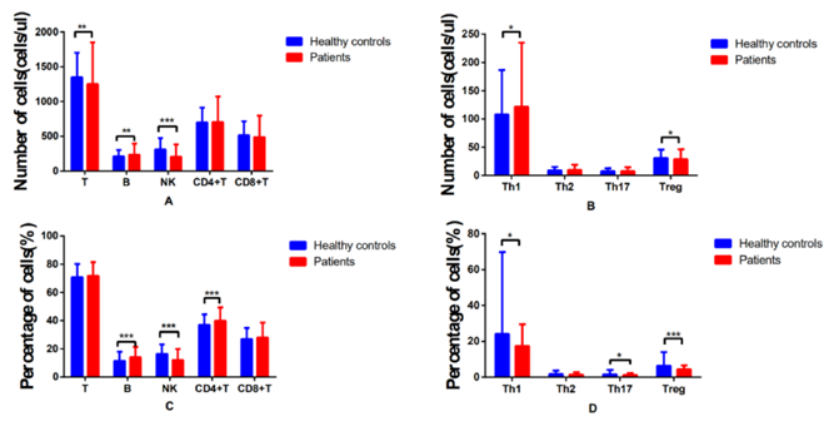

Figure 1: Comparison of peripheral blood lymphocyte subsets between healthy patients $(\mathrm{n}=206)$ and $\mathrm{pSS}$ patients $(\mathrm{n}=1221)$. All the lymphocytes subsets were analyzed by detected by flow cytometry combined with standard absolute counting beads. Data were presented as mean \pm SD and statistical analysis was determined by two-tailed unpaired t-test. $\left({ }^{*} \mathrm{P}<0.05,{ }^{* *} \mathrm{P}<0.01,{ }^{* * *} \mathrm{P}<0.001\right)$.

\section{References:}

[1] Mariette X, Criswell LA. Primary Sjogren's Syndrome. N Engl J Med 2018;378(10):931-39. doi: 10.1056/NEJMcp1702514 [published Online First: 2018/03/08]

[2] Miao M, Hao Z, Guo Y, et al. Short-term and low-dose IL-2 therapy restores the Th17/Treg balance in the peripheral blood of patients with primary Sjogren's syndrome. Ann Rheum Dis 2018;77(12):1838-40. doi: 10.1136/annrheumdis-2018-213036 [published Online First: 2018/06/25]

Acknowledgments : None.

Disclosure of Interests: None declared


Figure 2: Changes of peripheral lymphocyte subsets after immunoregulatory combination treatment $(\mathrm{n}=79)$. Data were expressed as mean \pm standard deviation to the distribution. Independent-samples $\mathrm{T}$ test and paired-samples $\mathrm{T}$ test were applied. $\mathrm{P}$ value $<0.05$ were considered statistically significant. ( $* P<0.05, * * P<0.01, * * * P<0.001)$

DOI: 10.1136/annrheumdis-2020-eular.2535

\begin{tabular}{l|l}
\hline SAT0184 & MAINTENANCE THERAPY WITH AZATHIOPRINE \\
ASSOCIATED WITH HIGHER RISK OF FLARE IN \\
PROLIFERATIVE LUPUS NEPHRITIS
\end{tabular}

M. Luis $^{1,2}$, A. R. Prata ${ }^{2}$, H. Assunção ${ }^{2}$, J. A. P. Da Silva ${ }^{1,3,4}$, L. Inês ${ }^{3,5} .{ }^{1}$ Faculty of Medicine, University of Coimbra, Coimbra, Portugal; ${ }^{2}$ Centro Hospitalar e Universitário de Coimbra, Rheumatology, Coimbra, Portugal; ${ }^{3}$ Centro Hospitalar e Universitário de Coimbra, Rheumatology, Coimbra, Portugal; ${ }^{4}$ Coimbra Institute for Clinical and Biomedical Research (iCBR), Coimbra, Portugal; ${ }^{5}$ School of Health Sciences, University of Beira Interior, Covilhã, Portugal

Background: Goals of lupus nephritis (LN) maintenance treatment include prevention of LN flares and long-term preservation of renal function, while minimizing drug iatrogenicity. There is an unmet need for identifying predictors of LN flare in order to guide optimization of maintenance immunosuppression.

Objectives: To identify predictors of $\mathrm{LN}$ flare after attainment of complete renal response $(C R R)$ in patients with proliferative $L N$.

Methods: Retrospective cohort study over 36 months including patients with SLE fulfilling the ACR'97 and/or the SLICC'12 classification criteria, enrolled in the CHUC Lupus Cohort between 1999 and 2018, with a biopsy-proven proliferative LN (class III/IV) and who attained CRR (proteinuria $<0.5 \mathrm{~g} /$ day and normal renal function, according to EULAR/ERA-EDTA definition) following induction treatment. Only proteinuric flares were considered and defined as doubling of proteinuria to $>1 \mathrm{~g} /$ day. Clinical-analytic characteristics at baseline (time of first CRR attainment after induction) were compared using survival analysis for time-to-flare. Variables with $p<0.10$ on univariate analysis with Log-Rank tests were further evaluated as predictors with multivariate Cox proportional hazards regression models (Backward Stepwise method, Wald-based), with estimation of hazard ratios (HR) with $95 \%$ confidence intervals $(95 \% \mathrm{Cl})$.

Results: A total of 50 patients in CRR were included in the analysis $(78.4 \%$ female, age at baseline $30.0 \pm 12.5$ years-old). Over the follow-up period, 10 patients $(20.0 \%)$ experienced a proteinuric flare, within a mean time of 29.1 months $(95 \% \mathrm{Cl} 26.89-31.37)$. In univariate analysis, age $<30$ years $(p=0.020)$, arterial hypertension $(p=0.020)$ and presence of anti-RNP antibody $(p=0.002)$ at baseline were associated with higher risk of LN proteinuric flares. In multivariate analysis, age $<30$ years (HR 26.56; $95 \% \mathrm{Cl} 1.93-365.08 ; \mathrm{p}=0.014$ ), arterial hypertension ( $\mathrm{HR} 8.30 ; 95 \% \mathrm{Cl} 1.21-56.92 ; \mathrm{p}=0.031$ ), use of antihypertensive antiproteinuric drugs (angiotensin-converting-enzyme inhibitors or angiotensin receptor blockers) (HR 11.18; 95\% $\mathrm{Cl} 1.24-100.66 ; p=0.031$ ) and maintenance therapy with azathioprine (HR 6.23; $95 \% \mathrm{Cl} 1.51-25.66 ; \mathrm{p}=0.011$ ) (Figure 1 ) were predictors of LN proteinuric flares. 